\title{
Comparação entre Resultados Analíticos e do Método dos Elementos de Contorno para o Problema de Transferência de Calor Bidimensional
}

\author{
Andrade, A. J. C $^{1^{*}}$; Loeffler, C. F. ${ }^{1}$ \\ 1 Programa de Pós-Graduação em Engenharia Mecânica, Universidade Federal do Espírito Santo, Vitória, ES, Brasil. \\ *e-mail: andrejudah@gmail.com
}

\begin{abstract}
Resumo
O método dos elementos de contorno apresenta bom desempenho em aplicações de campo escalar estacionário. Para a avaliação dos resultados numéricos, a forma mais eficaz é a comparação com os resultados analíticos da equação diferencial correspondente, nos casos onde a solução analítica é possível. Este trabalho compara os resultados numéricos e analíticos na resolução de um problema de transferência de calor com condição de contorno de Neumann. O programa computacional foi realizado em Fortran, utilizando o método dos elementos de contorno. Os resultados são avaliados em pontos no interior do domínio considerado, onde os erros apresentados foram bastante satisfatórios.
\end{abstract}

Palavras chaves: Método dos Elementos de Contorno, Solução Analítica, Transferência de Calor.

\begin{abstract}
The boundary element method performs well in aplications with steady state scalar field. The most effective way to evaluate the numerical results is compare it with the analytical results of the corresponding differential equation, if the analytic solution is possible. This paper compares the numerical and analytical results of a heat transfer problem with Neumann boundary condition. The program was developed in Fortran, using the boundary element method. The results are evaluated at interior points within the region of interest, where the calculated errors were satisfactory.
\end{abstract}

Keywords: Boundary Element Method, Analytical Solution, Heat Transfer.

\section{Introdução}

Diversos fenômenos da física e engenharias são modelados matematicamente através de Equações Diferenciais Parciais (EDP). Tais equações, mesmo respeitando simplificações consideráveis quanto a linearidade do comportamento de suas variáveis de estado e à uniformidade de suas propriedades constitutivas, entre outras hipóteses, frequentemente apresentam uma significativa complexidade.

$\mathrm{Na}$ atualidade, o rigor exigido na solução de fenômenos físicos de interesse à engenharia implica necessariamente na agilidade de obtenção de uma solução com precisão satisfatória. Em contraposição aos procedimentos analíticos de solução, que apresentam enormes restrições matemáticas para soluções simples e gerais, quanto às técnicas experimentais, que envolvem elevado custo e dispêndio de tempo (como no caso de protótipos e modelos em escala reduzida), as técnicas numéricas são efetivamente rápidas e flexíveis.

$\mathrm{Na}$ abordagem analítica dos problemas, alterações na conformação geométrica ou no tipo das condições de contorno aplicadas podem tornar sua solução inviável, mesmo se tratando de problemas escalares.

Não obstante as dificuldades da abordagem analítica e o êxito obtido pela simulação numérica, não se pode relegar a um plano secundário o procedimento de comparação das soluções numéricas com as correspondentes soluções matemáticas analíticas, particularmente quando problemas mais sofisticados são abordados ou então quando novas técnicas numéricas são desenvolvidas. Embora seja pertinente a comparação entre resultados provenientes de técnicas numéricas distintas, a forma mais precisa de avaliação dos resultados obtidos através de algum procedimento numérico é a comparação desses com resultados analíticos originários da solução da equação 
diferencial correspondente, nos casos onde a solução seja possível.

\section{Objetivo}

O objetivo deste trabalho é apresentar a comparação entre os valores numéricos e analíticos para um problema de transferência de calor bidimensional com condição de fluxo.

O valores analíticos são encontrados através da resolução da EDP característica do problema através do método da separação de variáveis. $E$ os resultados numéricos são obtidos através de programa em linguagem Fortran que utiliza o método dos elementos de contorno com interpolação direta (MECID), [1].

Uma vez que a solução analítica do problema de Laplace em duas dimensões é mais complicada e não é comum de ser encontrada na literatura especializada, um detalhamento da obtenção da mesma através do Método de Separação de Variáveis é apresentado.

Já para o Método dos Elementos de Contorno (MEC), sendo uma das mais importantes e conhecidas técnicas numéricas da atualidade, sua formulação é apresentada do modo mais simples possível.

\section{A Equação de Laplace}

Por vezes, muitos fenômenos apresentam similaridade quanto ao formato das equações características que os descrevem, sendo a equação de Laplace uma das mais conhecidas no campo dos fenômenos físicos que podem ser representados através de grandezas escalares. A Equação de Laplace é utilizada para modelar fenômenos que podem ser representados como funções de campo escalar, como escoamentos potenciais, processos de difusão, problemas de elasticidade [2]. Entre essas aplicações encontra-se a modelagem da transferência de calor em regime permanente [3], aplicação apresentada neste trabalho.

Assim, se o campo vetorial em regime permanente oriundo do gradiente de uma função escalar $T(x, y)$, neste caso representando a temperatura, apresenta seu divergente nulo em todos os pontos deste domínio, que se apresenta sem fontes ou sorvedouros, sua modelagem matemática é expressa por:

$$
\frac{\partial^{2} T}{\partial x^{2}}+\frac{\partial^{2} T}{\partial y^{2}}=\nabla^{2} T=0
$$

Para que o problema linear seja bem posto, é preciso apenas estabelecer condições de contorno. As condições de contorno mais comuns são de dois tipos: essenciais (condição de Dirichlet), quando os valores da função básica são conhecidos; naturais (condição de Neumann), quando suas derivadas com relação à normal exterior são prescritas em parte do contorno. Ambas são respectivamente expostas abaixo:

$$
\begin{aligned}
& \mathrm{T}(\mathrm{x}, \mathrm{y})=\overline{\mathrm{T}}(\mathrm{x}, \mathrm{y}) \text { prescrito em } \Gamma_{u} \\
& \mathrm{q}(\mathrm{x}, \mathrm{y})=\frac{\partial \mathrm{T}(\mathrm{x}, \mathrm{y})}{\partial \mathrm{n}}=\bar{q}(\mathrm{x}, \mathrm{y}) \text { prescrito em } \Gamma_{q}
\end{aligned}
$$

\section{O Método dos Elementos de Contorno}

O Método dos Elementos de Contorno se mostra como uma opção para a resolução de equações diferenciais em aplicações físicas. Diferente dos outros métodos de domínio (como Diferenças Finitas, Volumes Finitos e Elementos Finitos - MEF), o MEC se diferencia pela discretização do contorno, após o tratamento matemático através de formulação inversa [2], e resolução do sistema linear correspondente. Os valores internos ao contorno são interpolados em função dos resultados encontrados para os valores do contorno.

Assim, o ponto de partida tanto do MEF quanto do MEC pode ser feito, a partir da denominada forma integral forte associada à equação de Laplace:

$$
\int_{\Omega} \nabla^{2} \mathrm{Tw} \mathrm{d} \Omega=0
$$

A função w é uma função auxiliar e seu significado no MEC é a de ser a solução fundamental. Esta função é a solução de um problema correlato, governado por uma Equação de Poisson, onde uma fonte concentrada é aplicada num ponto específico de um meio infinito. Para se chegar a forma integral típica do MEC, é preciso usar o recurso matemático da integração por partes, chegando-se inicialmente à forma integral fraca associada, expressa a seguir:

$$
\int_{\Omega} \nabla \cdot(\nabla \mathrm{Tw}) \mathrm{d} \Omega=\int_{\Gamma}\left(\frac{\partial \mathrm{T}}{\partial \mathrm{x}_{\mathrm{j}}} \mathrm{w}\right) \frac{\partial \mathrm{x}_{\mathrm{j}}}{\partial \mathrm{n}} \mathrm{d} \Gamma=\int_{\Gamma} \mathrm{qw} \mathrm{d} \Gamma
$$

Nesta última equação foi empregado o Teorema da Divergência. Para se chegar ao ponto de partida do Método dos Elementos de Contorno, é preciso se proceder a mais uma integração por partes e novamente usar o Teorema da Divergência, chegandose a denominada forma inversa da equação integral:

$$
\int_{\Omega} \nabla^{2} \mathrm{w} \mathrm{T} d \Omega+\int_{\Gamma} \mathrm{qw} d \Gamma-\int_{\Gamma} \mathrm{T} \frac{\mathrm{dw}}{\mathrm{dn}} \mathrm{d} \Gamma=0
$$

No caso do MEC, consegue-se eliminar a integral de domínio que ainda persiste na equação anterior pelo fato da solução fundamental w ser a solução da seguinte equação diferencial:

$$
\frac{d^{2} w}{d r^{2}}+\frac{1}{r} \frac{d w}{d r}=-\Delta(\xi ; X)
$$

$\mathrm{Na}$ equação anterior $\xi$ é o ponto fonte, $\Delta$ representa a função Delta de Dirac e X consiste nas coordenadas de um ponto campo qualquer no domínio físico. Então, 


\section{Encontro Científico de Física Aplicada}

pode utilizando-se uma importante propriedade da função Delta de Dirac, tem-se:

$$
\mathrm{C}(\xi) \mathrm{T}(\xi)-\int_{\Gamma} q w \mathrm{~d} \Gamma+\int_{\Gamma} T \frac{\mathrm{dw}}{\mathrm{dn}} \mathrm{d} \Gamma=0
$$

C é uma constante que depende da localização do ponto $\xi$ em relação ao domínio $\Omega$. Através de um bem conhecido processo de discretização [4], gera-se um sistema matricial na forma:

$$
H T-G Q=0
$$

\section{Solução Analítica para o Problema de Transferência de Calor}

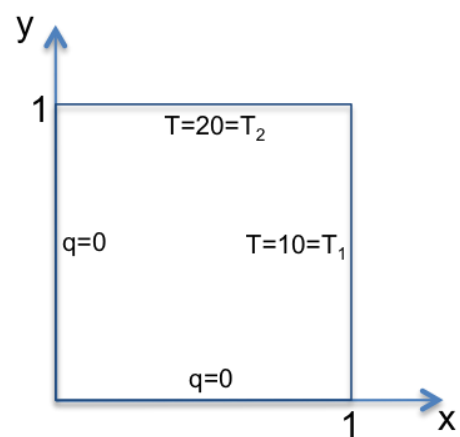

Figura 1: Domínio representado com as condições de contorno.

Para resolver analiticamente o problemas de Laplace usa-se aqui o Método da Separação das Variáveis (MSV). Esta técnica é uma das principais estratégias utilizadas para a obtenção de resultados analíticos para certas equações diferenciais parciais. Ela pode ser utilizada em casos específicos onde a forma da EDP permite a resolução da equação de forma analítica [5]. Diferentemente dos métodos numéricos discretos, sua implementação exige a descrição precisa da conformação geométrica do problema, bem como das condições de contorno envolvidas.

O domínio utilizado neste trabalho com as devidas condições de contorno (c.c.) é apresentado na Figura 1. Havendo condições de contorno não homogêneas, uma das formas de agilizar a solução via MSV consiste em realizar uma modificação na variável básica T:

$$
\theta=\frac{T-T_{1}}{T_{2}-T_{1}}
$$

Pelo MSV supõe-se uma solução do tipo:

$$
\theta(x, y)=X(x) Y(y)
$$

Substituindo na equação (11) e dividindo por XY:

$$
\frac{1}{Y} \frac{\partial^{2} Y}{\partial y^{2}}=-\frac{1}{X} \frac{\partial^{2} X}{\partial x^{2}}=\lambda^{2} \text { (uma constante) }
$$

Gerando 2 equações diferenciais ordinárias:

$$
\frac{d^{2} X}{d x^{2}}+\lambda^{2} X=0
$$

$$
\frac{d^{2} Y}{d y^{2}}-\lambda^{2} Y=0
$$

Sendo uma solução geral para equação (13) da forma:

$$
X=C e^{\gamma x}
$$

Substituindo em (13) e dividindo por $\mathrm{Ce}^{\gamma \mathrm{x}}$, surge:

$$
\gamma^{2}+\lambda^{2}=0
$$

Cuja solução para $\gamma$ é: $\gamma_{1,2}= \pm \lambda i$

Sobrepondo as soluções para $X$ e aplicando identidade trigonométrica, a equação (15) fica na seguinte forma:

$$
X(x)=C_{1} \cos (\lambda x)+C_{2} \operatorname{sen}(\lambda x)
$$

Fazendo o mesmo processo para $\mathrm{Y}$, supondo uma solução geral, a partir da equação (14) surge:

$$
\gamma^{2}-\lambda^{2}=0
$$

Cuja solução para $\gamma$ é: $\gamma_{1,2}= \pm \lambda$, de modo que:

$$
Y(y)=C_{3} e^{-\lambda y}+C_{4} e^{\lambda y}
$$

Utilizando as c.c em termos da nova variável $\theta$, podemos encontrar as constantes. Verificando que:

$$
\begin{aligned}
& \frac{\partial \theta}{\partial x}=Y \frac{\partial X}{\partial x}=Y\left(-\lambda C_{1} \operatorname{sen}(\lambda x)+\lambda C_{2} \cos (\lambda x)\right) \\
& \frac{\partial \theta}{\partial y}=X \frac{\partial Y}{\partial y}=X\left(-C_{3} \lambda e^{-\lambda y}+C_{4} \lambda e^{\lambda y}\right)
\end{aligned}
$$

Aplicando as condições naturais:

$$
\frac{\partial \theta}{\partial x}(0, y)=0=\left(C_{3} e^{\lambda y}+C_{4} e^{-\lambda y}\right) \lambda C_{2}
$$

Sendo $Y=C_{3} e^{-\lambda y}+C_{4} e^{\lambda y}$ diferente de zero, $C_{2}=0$.

$$
\frac{\partial \theta}{\partial y}(x, 0)=0=\left(C_{1} \cos (\lambda x)\right) \lambda\left(C_{4}-C_{3}\right)
$$

Sendo $X=C_{1} \cos (\lambda x)$ diferente de zero, $C_{3}=C_{4}$.

$$
\begin{aligned}
& \theta(x, y)=X(x) Y(y)=C_{1} \cos (\lambda x) C_{3}\left(e^{-\lambda y}+e^{\lambda y}\right)= \\
& D \cos (\lambda x) \cosh (\lambda y)
\end{aligned}
$$

Aplicando as condições essenciais, para $\theta(1, y)=0$ :

$$
\theta(1, y)=0=D \cos (\lambda) \cosh (\lambda y)
$$

Como $\cosh (\lambda y)>0, \cos (\lambda)=0$. Dessa forma, $\lambda=(2 n-1) \pi / 2$, $n=1,2,3 \ldots$

Superpondo as $n$ soluções e chamando $D$ de $C_{n}$ :

$$
\begin{aligned}
& \theta(x, y)=\sum_{n=1}^{\infty} C_{n} \cos \left((2 n-1) \frac{\pi}{2} x\right) \cosh ((2 n- \\
& \text { 1) } \left.\frac{\pi}{2} y\right)
\end{aligned}
$$


Para c.c. $\theta(x, 1)=1$ :

$$
\begin{aligned}
& \theta(x, 1)=1= \\
& \sum_{n=1}^{\infty} C_{n} \cos \left((2 n-1) \frac{\pi}{2} x\right) \cosh \left((2 n-1) \frac{\pi}{2}\right)
\end{aligned}
$$

Qualquer função $f(x)$ pode ser expressa em termos de séries infinitas de funções ortogonais [4]:

$$
f(x)=\sum_{n=1}^{\infty} A_{n} g_{n}(x)
$$

Assim, multiplicando ambos os lados da equação por $g_{n}(x)$ e integrando-se entre os limites $a$ e $b$, podemos encontrar os coeficientes $A_{n}$ :

$$
A_{n}=\frac{\int_{a}^{b} f(x) g_{n}(x) d x}{\int_{a}^{b} g_{n}^{2}(x) d x}
$$

Voltando a (27) e chamando:

$$
\begin{aligned}
& C_{n}=\frac{A_{n}}{\cosh \left((2 n-1) \frac{\pi}{2}\right)} \\
& \theta(x, 1)=1=\sum_{n=1}^{\infty} A_{n} \cos \left((2 n-1) \frac{\pi}{2} x\right)
\end{aligned}
$$

Escolhendo $f(x)=1$ e a função ortogonal $g_{n}(x)=\cos ((2 n-$ 1) $\pi x / 2)$, para o intervalo de $(0,1)$ e substituindo-as na equação (29) obtêm-se:

$$
\begin{aligned}
& A_{n}=\frac{\int_{0}^{1} \cos ((2 n-1) \pi x / 2) d x}{\int_{0}^{1} \cos ^{2}((2 n-1) \pi x / 2) d x}=\frac{4(-1)^{n+1}}{\pi(2 n-1)} \\
& C_{n}=\frac{4(-1)^{n+1}}{\pi(2 n-1)} \frac{1}{\cosh \left((2 n-1) \frac{\pi}{2}\right)}
\end{aligned}
$$

Assim, a solução fica:

$$
\begin{aligned}
& \theta(x, y)=\sum_{n=1}^{\infty} \frac{4(-1)^{n+1}}{\pi(2 n-1)} \frac{\cosh \left((2 n-1) \frac{\pi}{2} y\right)}{\cosh \left((2 n-1) \frac{\pi}{2}\right)} \cos ((2 n- \\
& \text { 1) } \left.\frac{\pi}{2} x\right)
\end{aligned}
$$

Retornando em termos da variável original:

$$
\begin{aligned}
& T(x, y)=\left(\sum_{n=1}^{\infty} \frac{4(-1)^{n+1}}{\pi(2 n-1)} \frac{\cosh \left((2 n-1) \frac{\pi}{2} y\right)}{\cosh \left((2 n-1) \frac{\pi}{2}\right)} \cos ((2 n-\right. \\
& \text { 1) } \left.\left.\frac{\pi}{2} x\right)\right)\left(T_{2}-T_{1}\right)+T_{1}
\end{aligned}
$$

\section{Comparação de Resultados}

Aplicando a geometria e as condições de contorno ao programa de MEC, são gerados resultados para uma discretização com 16 elementos lineares. Esses resultados são comparados com os valores analíticos da equação (35), para $n=15$, em diferentes pontos internos no domínio. A tabela 1 apresenta a comparação dos resultados.

Tabela 1: Resultados analíticos e numéricos comparados em 6 pontos do domínio.

\begin{tabular}{ccccc}
\hline $\mathbf{x}$ & $\mathbf{y}$ & $\begin{array}{c}\mathbf{T}- \\
\text { Programa }\end{array}$ & $\begin{array}{c}\mathbf{T}- \\
\text { Analítico }\end{array}$ & Erro (\%) \\
\hline 0.12 & 0.50 & 16.3119 & 16.2874 & $0.1504 \%$ \\
\hline 0.30 & 0.22 & 14.7662 & 14.7713 & $0.0343 \%$ \\
\hline 0.64 & 0.84 & 17.3672 & 17.3264 & $0.2356 \%$ \\
\hline 0.90 & 0.50 & 11.2647 & 11.2793 & $0.1291 \%$ \\
\hline 0.70 & 0.70 & 15.0000 & 15.0000 & $0.0000 \%$ \\
\hline 0.50 & 0.20 & 13.8258 & 13.8426 & $0.1211 \%$ \\
\hline
\end{tabular}

Os resultados apresentados foram considerados satisfatórios. O erro para o modelo numérico ficou menor que $0,24 \%$ para os pontos analisados.

\section{Conclusão}

Os resultados apresentados neste trabalho demonstraram a comparação entre os resultados do método dos elementos de contorno com a solução analítica de um problema proposto.

A principal dificuldade em experimentos numéricos é a disponibilidade de soluções analíticas, ou soluções reconhecidamente robustas para a verificação dos resultados, algo que pode ser realizado neste trabalho.

\section{Referências}

[1] LOEFFLER, C. F.; CRUZ, A. L.; BULCÃO, A. Direct use of radial basis interpolation functions for modelling source terms with the boundary element method. Engineering Analysis with Boundary Elements, v. 50, p. 97-108, 2015.

[2] BREBBIA, C. A.; DOMINGUEZ, J. Boundary elements: an introductory course. WIT press: 1996.

[3] INCROPERA, F. P.; DEWITT, D. P. Fundamentals of heat and mass transfer. John Wiley \& Sons: 2011.

[4] BREBBIA, C. A. The boundary element method for engineers. London: Pentech Press: 1978.

[5] SAGAN, H. Boundary and Eigenvalue Problems in Mathematical Physics. New York: John Wyley \& Sons: 1963. 